\title{
Wireless Network Simulation in Aircraft Cabins
}

\author{
Mennatoallah Youssef *, Student Member, Old Dominion University, Norfolk, Virginia \\ Linda Vahala, Senior Member, Old Dominion University, Norfolk, Virginia \\ John H. Beggs, Senior Member, NASA Langley Research Center, Hampton, Virginia
}

\begin{abstract}
An electromagnetic propagation prediction tool was used to predict electromagnetic field strength inside airplane cabins. A commercial software package, Wireless Insite ${ }^{\circledR}$, was used to predict power levels inside aircraft cabins and the data was compared with previously collected experimental data. It was concluded that the software could qualitatively predict electromagnetic propagation inside the aircraft cabin environment.
\end{abstract}

\section{INTRODUCTION}

In today's industrialized world, wireless computer networking is becoming more prevalent. Wireless computer networks are of significant interest to the aviation industry for enhanced passenger connectivity. Wired network systems are unattractive due to significant additional space, weight, and maintenance costs, in addition to very high retrofit costs. Wireless networks are an obvious choice due to much lower space, weight and maintenance requirements and much simpler retrofit and installation.

The main disadvantage of physically testing a wireless network in an airplane fuselage is the time and cost involved. It is cost prohibitive to physically test each airplane in the commercial fleet that may be retrofitted with a wireless network. Modeling and simulation tools could be applied to various classes of airplanes to provide a general idea of wireless network design and optimum placement of network components (e.g. access points). To overcome this particular challenge, an electromagnetic propagation prediction tool can be used to simulate the environment in an aircraft cabin. Modeling and simulation decrease the time and cost associated with physical testing, and they can also provide quick parameter changes on demand. The focus of the present work is to determine whether commercial software (Wireless Insite (WI)) by Remcom, Inc. can be an effective and accurate method of simulating wireless communication within aircraft cabins.

\section{METHOD}

To validate EM power propagation predictions inside aircraft cabins, it is necessary to compare the simulation to an experimental study. Embry-Riddle Aeronautical University tested WLAN (Wireless Local Area Networks) performance in four airplanes (B747-400, B767-300, B777-200, \& Airbus A320-200) [1]. The parameters of the experimental study were taken into account when building the simulation. Three different access points (APs) were used in the experimental study to transmit $801.11 \mathrm{a}$ and $802.11 \mathrm{~b}$ signals. Specifically, the ORiNOCO AP 2000 is the AP simulated in the present work. The experimental test results indicated that this AP most complied with standards set in the experimental study.

Table I Parameters of the Access Points

\begin{tabular}{l|l|l|l}
\hline \hline Access Points & ORiNOCO AP 2000 & LinkSys WAP51AB & NetGear WG-602 \\
\hline Standard & $802.11 \mathrm{a} \& 802.11 \mathrm{~b}$ & $802.11 \mathrm{a} \& 802.11 \mathrm{~b}$ & $802.11 \mathrm{~b} \& 802.11 \mathrm{~g}$ \\
Antenna Type & Two omnidirectional & Single linear dipole & $1 / 2$ wave dipole \\
Propagation & Vertical & Vertical & Vertical \\
Antenna Gain & $5.0 \mathrm{dBi}$ & $1.0 \mathrm{dBi}$ & $2.0 \mathrm{dBi}$ \\
Output Power & $802.11 \mathrm{a} 17 \mathrm{dBm}$ & $802.11 \mathrm{a} 14.653 \mathrm{dBm}$ & $802.11 \mathrm{~b} 10.530 \mathrm{dBm}$ \\
& $802.11 \mathrm{~b} 18 \mathrm{dBm}$ & $802.11 \mathrm{~b} 10.530 \mathrm{dBm}$ & $802.11 \mathrm{~g} 10.530 \mathrm{dBm}$ \\
Polarization & Vertical & Vertical & Vertical \\
Transmission Line loss & -2.0 & -2.0 & -2.0 \\
\hline \hline
\end{tabular}


Each airplane had a distinct location of APs and wireless receivers. These are referenced from 802.11 Wireless Network Performances within Aircraft Cabins [1]. Before the experimental attributes can be added to the simulated environment, the aircraft cabins must be modeled. The accuracy and relevance of the simulation greatly depends on the accuracy put forth in recreating the cabin geometry. The first fundamental step is to create the cabin in WI. The most efficient way to build the cabin model was to import data exchange files (.dxf), which were created in an AUTOCAD capable program. The complex nature of the fuselage makes it difficult to model with software intended for simpler structures such as offices and buildings. As mentioned above, the Boeing 747-400, 767-300, 777-200, and the Airbus A320 were the specific models used. Two different cabin representations (a rectangular prism and a half-cylinder) were used in the simulation, and no internal cabin details such as windows, galleys, doors, seats or overhead cargo bins were included. The rectangular prism had the same cabin cross sectional area as the four aircraft cabins mentioned above. All cabins have two models except for the B747-400. This cabin has two levels and the experimental measurements were collected on the lower level. Thus the $747-400$ cabin geometry was a rectangular shape with a front-end taper. A second floor was also added since it will couple energy from the power propagation. These simple cabin models should provide some general insight into the software validity. More complex models with internal components can be created later to examine their effect on power propagation [1].

(a)

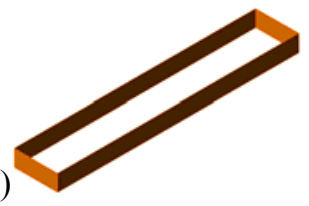

(c)

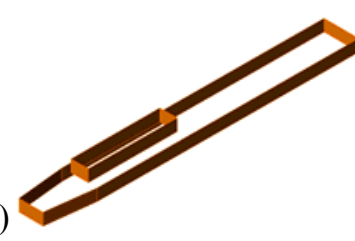

(b)

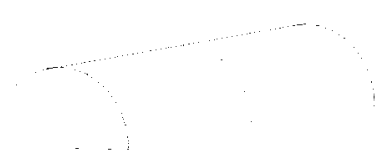

(d)

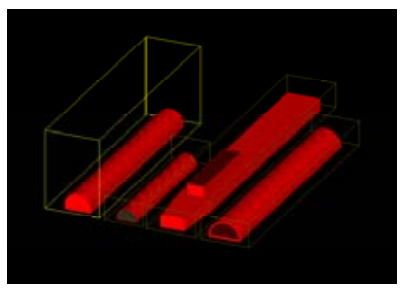

Figure I: (a) Rectangular Representation (b) Imported CAD Drawing (c) Boeing 747 fuselage (d) Visualization in Wireless Insite

After the AutoCAD drawings were imported, they were assigned material properties. For simulation purposes, we assumed that the fuselage metal was a Perfect Electrical Conductor (PEC). Various coefficient types such as roughness, conductivity, permittivity, and thickness are also incorporated [2].

It was important to place the transmitters and receiver in the same location and orientation as the experimental setup. Transmitters needed the following properties to operate: coordinate system elevation type, rotation, waveform, antenna type, radiated power, and rotation about each plane in the xyz system. The height, rotation about the axis, and orientation for the transmitters differed for each plane. Receivers are much easier to place. The receivers were laid as a strip on the left portion of the fuselage (as shown in Figure II). The receiver extended from the transmitter to the aft end of the fuselage. Ideally the receiver can be placed on an airplane tray or the seat.

Four different antennas were needed to model the $802.11 \mathrm{a}$ and $802.11 \mathrm{~b}$ standards. For the transmitters, a linear dipole antenna was used for both, with the individualized waveform, antenna length, gain, and transmission line loss. The WI database provided the antenna basics for the transmitter. WI also generated the antenna patterns. The receiver antennas were not generated by the WI database since they are integrated antennas. An antenna format file was created from the antenna pattern of the receiver. The antenna was then imported and assigned to the receiver. 
WI also provides various propagation methods for the user. Three different methods of propagation were considered for simulation (Urban Canyon, Fast 3D \& Full 3D). All three methods were used to predict data. Two of the three methods use approximations to produce data; the third method is expected to be the most accurate, but takes longer to run. We must determine if the approximations made by the methods are adequate for our geometries. Only one of the methods is recommended for indoor use. But since the cabin has a unique shape, the indoor method may not be the most accurate [2].

The Fast 3D Model (FM) results are the emphasis of the present work. This method is used when transmitters and receivers are close to the ground in height as compared to the height of other objects. The FM model uses the shooting and bouncing ray method (SBR) to determine the propagation paths of the transmitters. The ray tracing method uses SBR for horizontal plane and an image method (IM) for ground reflections. The FM does not use a full 3D trace; rather, Fast 3D means that the vertical components of some objects are considered in the calculations. FM incorporates the heights of other objects when making calculations. It is generally used if there are many objects of varying heights that could possibly have ray paths to affect the calculations.

\section{RESULTS}

The results of the simulation are graphed along with the experimental data in Figure III. The simulated results were exported and the standard deviation between the points was calculated and is shown in Table II. Station number on the graph indicates the location of the receiver points and the received power is shown in Figure II as a color map and graphically in Figure III. The client card measurements are experimental data collected using a wireless client card inserted into a laptop computer. The experimental spectrum analyzer data was collected using an uncalibrated antenna. It is shown in the graph to demonstrate how the predicted data falls within the range of the spectrum analyzer and the client card data.

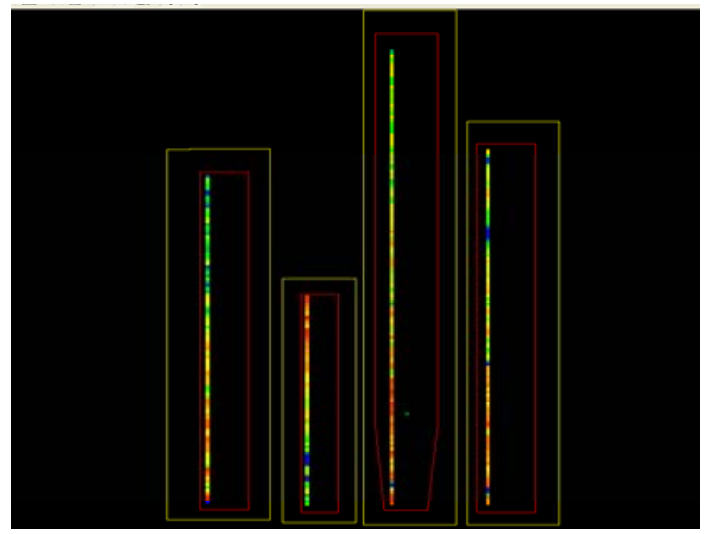

Figure II: Power outputs visually depicted in Wireless Insite for the four fuselages. Each color represents outputted power and the receiver location. This is a topical view of Figure $1 d$. 
Figure III: Comparison of Measured Power to simulation of a Boeing767 with Orinoco B specifications with FAST 3D Method of Simulation

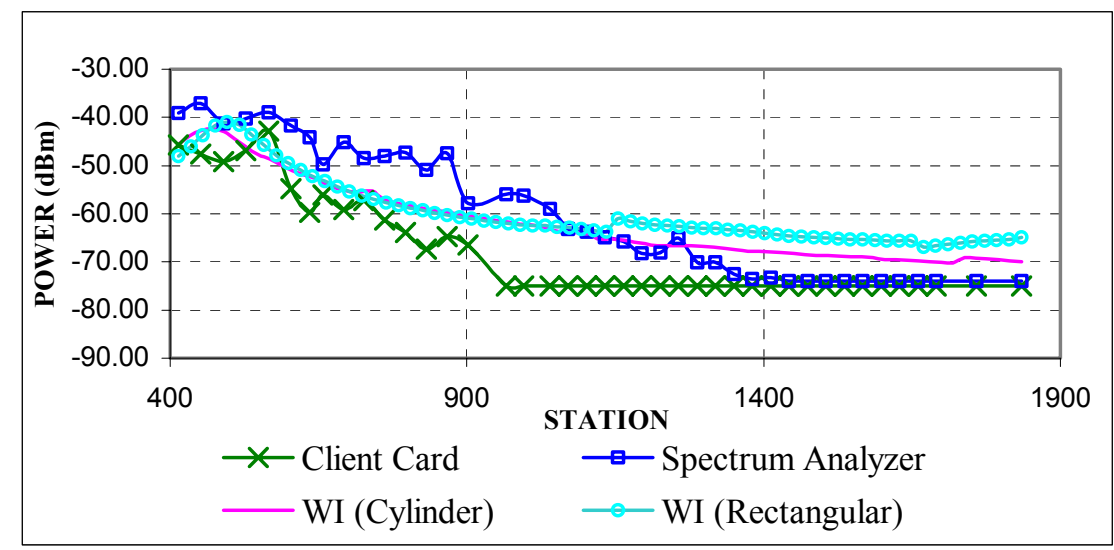

Table II: Standard Deviations as compared to the Spectrum Analyzer using the Fast 3D Method

\begin{tabular}{lccc}
\hline \hline Aircraft & Rectangle & Half-Cylinder & Standard \\
\hline B747 & 6.1 & N/A & $802.11 \mathrm{a}$ \\
B747 & 4.3 & N/A & $802.11 \mathrm{~b}$ \\
B767 & 7.4 & 6.7 & $802.11 \mathrm{a}$ \\
B767 & 5.8 & 3.1 & $802.11 \mathrm{~b}$ \\
B777 & 10.1 & 6.1 & $802.11 \mathrm{a}$ \\
B777 & 11.4 & 4.9 & $802.11 \mathrm{~b}$ \\
A320 & 10.0 & 7.0 & $802.11 \mathrm{a}$ \\
A320 & 6.9 & 6.0 & $802.11 \mathrm{~b}$ \\
\hline \hline
\end{tabular}

When examining the data in Table II, one trend becomes apparent; the rectangular model is slightly less accurate than the cylindrical model when compared to the experimental data. In Table II, it was noted that the rectangular model had a slightly higher standard deviation in each instance. In Figure III, the predicted results are within about $10 \mathrm{dBm}$ over the entire length of the aircraft cabin.

The qualitative performance of the predicted data is quite good relative to the experimental spectrum analyzer and client card data. It should also be noted that the predicted results are much smoother than the experimental data. This is because the experimental setups included the internal components of the in-service aircraft cabins, such as seats, tray tables, armrests, overhead cargo bins, galleys and lavatories. The predicted data follows the same general trend throughout the cabin as the experimental data, and it almost appears to be an average between the client card data and spectrum analyzer data.

\section{CONCLUSION}

The goal of the present work was to determine if commercial off-the-shelf electromagnetic modeling software could be used to predict electromagnetic propagation inside aircraft cabins. The results clearly demonstrate that this is possible and quite feasible, and the predictions give good qualitative agreement with experimental data. Furthermore, the level of agreement was surprising, given the relative crudeness of the initial aircraft cabin models. More accurate results are anticipated with further studies and refinements of the aircraft cabin geometries.

\section{REFERENCES}

1. Whetten, F.; Soroker, A.; Whetten, D. 802.11 Wireless Network Performances within Aircraft Cabins. Embry-Riddle Aeronautical University: 2003.

2. Remcom Incorporated. Wireless Insite User's Manual 1.5.0 October 2003 